\title{
Dried urine swabs as a tool for monitoring metabolite excretion
}

\author{
Rastislav Monošík*,1 \& Lars Ove Dragsted ${ }^{1}$ \\ ${ }^{1}$ Department of Nutrition, Exercise \& Sports, Faculty of Life Science, University of Copenhagen, Nørre Allé 51, DK-2200 \\ Copenhagen N, Denmark \\ *Author for correspondence: ram@nexs.ku.dk
}

\begin{abstract}
Aim: We tested a large set $(n=181)$ of wet urine and dried urine samples spotted on regular cosmetic cotton swabs for comparative UHPLC-MS/MS analysis of various metabolites across a wide polarity and structural range. Results/methodology: The agreement of measurements between conventional $24 \mathrm{~h}$ urines and dried urine spots made from them in situ was evaluated by Passing-Bablok regression and BlandAltman analysis after creatinine correction. There was full agreement in qualitative results but quantitative analysis revealed underestimation of dried urine spots in some cases. The dried urine samples contained analytes at measurable levels for at least 9 months. Conclusion: Although this technique seems very promising more methodological studies have to be conducted in order to improve applicability of dried urine microvolume fluidic sampling for quantitative analysis.
\end{abstract}

First draft submitted: 12 February 2018; Accepted for publication: 11 July 2018; Published online: 8 August 2018

Keywords: creatinine correction • dried urine spots $\bullet$ UHPLC-MS/MS

Repeated urine sampling throughout the study period increases the number of dietary-related metabolites measured and therefore the coverage of dietary markers of food intake in nutritional trials. However, sample collection and the number of volunteer visits in the clinic can be very demanding and costly. Less demanding sampling techniques easing the sampling procedure (especially for blood and urine), decreasing the burden to volunteers, and lowering the costs should be achieved while providing documented storage stability and reliable results. A promising approach for this purpose is to spot samples on a carrier and letting it dry. Dried blood spots (DBS) or urine spots (DUS) can be collected, temporarily stored and transferred by the volunteers themselves at a convenient time. Around 100-times as many research papers exist for DBS compared with DUS which is documented by 4654 search results for ([dry OR dried] AND 'blood spot*') versus 45 results for ([dry OR dried] AND 'urine spot*') (on 6 June 2018 in Web of Science ${ }^{T M}$ using 'Topic' as search field); DBS also has its own commercial platform and database [1]. Nevertheless, DUS could become the method of choice if an analyte can be measured in both matrices since DBS suffers from hematocrit influence on spot size, recovery, blood-to-plasma ratio [2] and cannot be considered as a fully noninvasive approach. However, for quantitatively accurate analysis fluctuation in urine volumes must be taken into account and corrected. DUS samples on filter paper have previously been successfully tested for various applications, such as diagnosis of glutaric aciduria type 1 [3], detection and quantification of hippuric acid [4], creatinine [4-6], sulfatide molecular species [7], cytomegalovirus [8], several drug classes [9], opioids [10], drugs of abuse [11], etc. Nevertheless, much investigation is still required in the field of dry spot sampling for it to become an accepted methodology for quantitative analysis [12].

In this study, we tested the possibility of using cotton swab kits produced in-house for collection of DUS instead of spotting them on a filter paper, which has been so far the method of choice [13,14]. Based on our experience, urine swabs as presented in this work are easier to use for subjects than DBS and more straightforward to obtain than DUS on paper cards $[15,16$. Developing cotton swabs additionally provided the possibility of direct extraction, while DUS on paper cards usually requires punching of discs before extraction $[17,18]$. This difference could be particularly important in trials with a high number of collected samples.

The aim of this study was primarily to understand the potential of using DUS on cosmetic swabs for metabolite profiling and to test the agreement of qualitative and quantitative data obtained by LC-MS/MS analysis [19] 
compared with wet $24 \mathrm{~h}$ urine samples after creatinine adjustment. Second, we investigated intrapersonal variance of creatinine levels over a period of 6 weeks in order to investigate whether total daily creatinine excretion is constant enough to be useful for determining an individual average correction factor based on a few measurements in future longitudinal trials. To the best of our knowledge, this is the first comparative study of $24 \mathrm{~h}$ urine samples versus extracts made on dried urine collections for quantitative simultaneous analysis of several biomarkers across a wide polarity and structural range (possible application of these biomarkers are indicated in reference [19]). Samples were extracted up to 9 months after collection which has been the longest storage period tested so far.

\section{Experimental}

\section{Chemicals \& materials}

Opaque plastic tubes $(11 \mathrm{~cm})$ with desiccant filled stoppers were obtained from Clariant Healthcare Packaging (Romorantin, France). Silica gel orange granular, 0.2-1 mm, was purchased from Sigma-Aldrich (Schnelldorf, Germany). All other chemicals and materials used for analysis were obtained as described in our earlier work [19].

\section{$24 \mathrm{~h}$ urine \& DUS sample preparation}

The study protocol was approved by the municipal Ethical Committee of the capital region of Denmark (journal number: H-15016961) in accordance with the Helsinki-II declaration. The study was registered on Clinicaltrials .gov (NCT03384147). Before entering the study all participants gave their written consent after having received written and oral information about the study. Twenty-six subjects provided 181 total volume $24 \mathrm{~h}$ urine samples. Each subject provided seven samples and only in one instance the sample was not delivered. Samples were collected from healthy men and women in the age range 20-70 years, excluding binge drinking subjects, subjects with known past or present chronic diseases, a history of substance abuse or at increased risk of breast cancer. All $24 \mathrm{~h}$ urine samples were collected in cooled $\left(5-7^{\circ} \mathrm{C}\right) 21$ polypropylene plastic containers (Grathwol, Karlslunde, Denmark) and defined as a collection starting with the second urine void in a day and ending with the first void (included) on the next day. At delivery, all urine samples were anonymized by coding, weighed, measured for density to calculate volume, pipetted (Biohit pipettes, Sartorius-Dandiag, Ishoj, Denmark) into cryotubes, and stored at $-80^{\circ} \mathrm{C}$ until the day of analysis.

DUS analog samples were prepared in situ using regular commercially available cosmetic cotton swabs (Matas, Copenhagen, Denmark) on the same day as $24 \mathrm{~h}$ urine was received. The swabs were composed of a plastic handle and cotton head (no technical specifications were provided on the package). Pasteur pipettes were used to apply a few drops of each well-mixed $24 \mathrm{~h}$ urine on both cotton ends of the stick until fully soaked. The applied volume was estimated to be in the range $80-120 \mu \mathrm{l}$. Swabs were then inserted into a plastic tube, closed with a desiccant-filled stopper, and stored vertically at room temperature until the day of analysis. The extraction of the samples was performed 5 months after the last $24 \mathrm{~h}$ urine sample was obtained, therefore the storage time of the DUS varied from 5 to 9 months. A DUS kit prototype produced in-house for self-sampling by volunteers tested in the long-term storage-trial is illustrated in Supplementary Figure 1.

\section{Creatinine analysis}

Creatinine concentrations were analyzed in $24 \mathrm{~h}$ urines and in DUS extracts by the standard bioanalytical assay using ABX Pentra 400 (Horiba ABX SAS, France). Quality control was performed by repeated analysis of diluted and highly concentrated control samples delivered together with the kit: creatinine 120 CP (Lot number 1603401 and 212286416). The lower limit of quantification of this assay was determined by the manufacturer according to the CLSI (NCCLS), EP17-A protocol, and equals $261 \mu \mathrm{mol} \mathrm{l}^{-1}\left(2.9 \mathrm{mg} \mathrm{dl}^{-1}\right)$ [20].

\section{Experimental setup for creatinine correction to calculate excreted amounts}

A schematic overview of the experimental setup to compare $24 \mathrm{~h}$ urine and DUS total analyte excretion is shown in Figure 1. For $24 \mathrm{~h}$ urine this is simply the sample concentration measured, multiplied by $24 \mathrm{~h}$ urine excretion volume. $24 \mathrm{~h}$ urine contains total daily excretion of any target metabolite ' $\mathrm{M}$ ' and of creatinine ' $\mathrm{C}$ ', while the extract from the cotton swab only contains a portion of the metabolite ' $m$ ' and creatinine ' $c$ '. The ratio of $\mathrm{C}$ and c provides the correction factor ' $\mathrm{f}$ ' and the relationship between measured parameters can be characterized by the equation as presented in Figure 1. 
Figure 1. A schematic overview of the experimental setup. $24 \mathrm{~h}$ urine sample aliquots were diluted $10 x$ with water containing $5 \% \mathrm{MeOH}$ ( $\mathrm{v} / \mathrm{v})$ and internal standards prior to UHPLC-MS/MS analysis. Total metabolite ' $M$ ' and creatinine ' $C$ ' excretion was calculated using the known total urine volume. The DUS was extracted in $1 \mathrm{ml}$ of milli-Q water in $15 \mathrm{~min}$ and fractional daily metabolite excretion ' $m$ ' was measured by the same UPLC-MS/MS method, while fractional creatinine ' $c$ ' excretion was measured using a commercial kit. The relationship between ' $M$ ' and ' $m$ ' using correction ratio ' $\mathrm{C} / \mathrm{C}$ ' is expressed by the equation.

DUS: Dried urine swab.

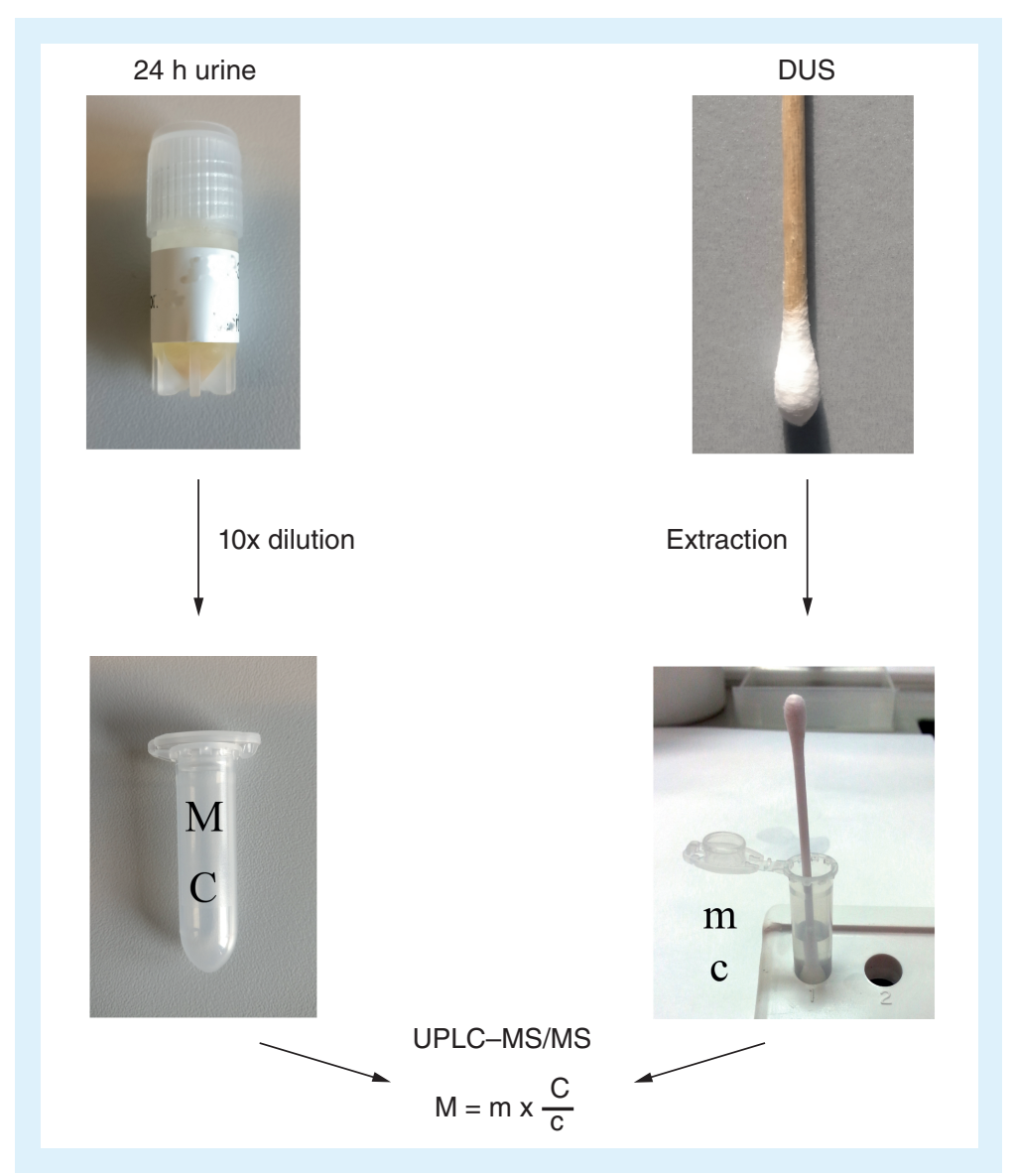

\section{UHPLC-MS/MS analysis}

The urine samples were thawed on ice, shaken and centrifuged at 2400 r.p.m. (1210 rcf) for 3 min and $20 \mu l$ of the supernatant was $10 \times$ diluted in water containing $5 \%$ methanol $(\mathrm{v} / \mathrm{v})$ and the internal standards prior to UHPLC-MS/MS analysis.

DUS samples were randomized, cut in two pieces and alternately either upper or bottom cotton swab (relative to the cap with desiccant) was extracted in $1 \mathrm{ml}$ of milli-Q water in an Eppendorf tube for $15 \mathrm{~min}$. Swab position was not taken into account in the analysis. Milli-Q water was chosen as a DUS extraction agent since it allows analyzing creatinine in the extract on automated analyzers without jeopardizing hardware components sensitive to organic solvents. Of this extract $190 \mu \mathrm{l}$ were mixed with $10 \mu \mathrm{l}$ of water containing $5 \%$ methanol $(\mathrm{v} / \mathrm{v})$ and the internal standards prior to UHPLC-MS/MS analysis. An overview of the gradient steps in the UHPLC (Waters Acquity H-class, Manchester, UK) analytical method is presented in Supplementary Table 1. A Waters ACQUITY UPLC ${ }^{\circledR}$ HSS T3 $100 \AA$ pore size, $1.8 \mu \mathrm{m}$ particle size, $2.1 \mathrm{~mm} ø \times 100 \mathrm{~mm}$ column (CT, USA) was used at a temperature of $40^{\circ} \mathrm{C}$.

Tartaric acid (TaA), ethyl sulfate (EtS), ethyl glucuronide (EtG), indoxyl sulfate ( $\mathrm{InS})$, cresol sulfate (CrS), and dehydroepiandrosterone sulfate (DHEAS) were analyzed according to a validated method described in detail previously [19]. Due to method transfer to Waters ACQUITY UPLC H-Class System ${ }^{\circledR}$ (CT, USA) using quaternary solvent configuration, minor modifications to the flow rate and gradient steps to readjust retention times were made (see Supplementary Table 1 for details). Additionally, multiple reaction monitoring transitions for pyroglutamyl proline (PgP) were added to the method based on our recent research [21,22]. Since PgP standards were not available, 3-nitro tyrosine together with 3-nitro tyrosine- $\mathrm{d}_{3}$ were used as calibration and internal standards, respectively; this correction is of course arbitrary but should not affect the comparative analyses. As shown in Supplementary Figures 2 and $3, \mathrm{PgP}$ shares most of the fragments at collision energy $\geq 20 \mathrm{~V}$ and has a comparable retention time to 3-nitro-tyrosine (Supplementary Figure 4). It is assumed that possible difference in fragmentation efficiency of $3-\mathrm{NT}$ and PgP could have affected true values (for all samples) but not the comparative agreement of $24 \mathrm{~h}$ urine 
versus DUS using the same calibrants. All multiple reaction monitoring transitions with the detector settings are summarized in Supplementary Table 2.

Subjects and sample injection order were randomized using the 'RAND()' function in Microsoft Excel and all samples from any particular subject were analyzed on the same plate. Each of the six analyzed plates (batches) contained standards for the calibration curve $(n \geq 6)$. For quality control each plate also contained a batch pool and a total pool of all urine samples with and without spiking, injected after every 10 study samples. Spiking of the pools was done by adding a calibrant mixture, selecting the mid-range concentration within the observed linear range for each compound. This was done in order to cover quality control also for samples with higher concentrations of the analytes and to cover compounds present in the unspiked pools at concentrations below the LLOQ. Plates were analyzed in random order within 6 days.

\section{Relative extraction recovery, repeatability \& stability of DUS}

Relative extraction recovery (i.e., comparing DUS recovery with that from intact urine, which in itself is not guaranteed to give $100 \%$ recovery) and repeatability of DUS experiments were examined by pipetting $80 \mu \mathrm{l}$ of randomly selected $24 \mathrm{~h}$ urine samples onto cotton swabs. Seven different urine samples were used for making DUS and the first three of them were made in duplicates. The seven DUS were produced in order to examine the relative extraction recovery efficiency in different urine matrices, while the three additional DUS were made to examine the effect of extraction time. These DUS were dried for $2.5 \mathrm{~h}$ in a closed in-house prepared can with a silica gel (Supplementary Figure 5). The set of seven DUS were afterward extracted for 15-20 min, while the duplicates of the first three DUS were extracted for 60 min.

A pool of the seven samples was additionally prepared and used to make two sets of triplicate DUS. The first set was extracted immediately without drying and the other was extracted after $2.5 \mathrm{~h}$ of drying in the can with silica.

An independent set of eight replicates of DUS made from a randomly selected urine sample were dried by a gentle $\mathrm{N}_{2}$ stream and extracted for $20 \mathrm{~min}$ in $1 \mathrm{ml}$ of milli-Q water for determination of the repeatability of relative extraction recovery. Immediately after removal from the first (water) extraction these DUS underwent also a second extraction in $1 \mathrm{ml}$ of $20 \%$ methanol (v/v) in order to investigate possible unextracted analytes. The volume left on the stick (carry-over) was around $10 \%$ (average $100 \mu \mathrm{l})$.

The selected metabolites (TaA, EtS, EtG, InS, CrS, PgP and DHEAS) were quantified by UHPLC-MS/MS, and creatinine on a Pentra analyzer. This was done both for original (wet) urine samples and for their corresponding DUS extracts. Relative recovery of metabolites without creatinine correction taking into account urine $(10 \times)$ and theoretical DUS dilution $(1000 / 80 \times 200 / 190=13.16$-fold for UHPLC-MS $/$ MS analysis and 1000/80 = 12.5fold for creatinine Pentra analysis) was calculated according to the formulas:

UHPLC - MSMS relative recovery $\%=\left(\mathrm{c}_{\text {DUS }} \times 13.16 / \mathrm{c}_{\text {urine }} \times 10\right) \times 100$, and

Pentra relative recovery $\%=\left(c_{\text {DUS }} \times 12.5 / c_{\text {urine }} \times 10\right) \times 100$

Long-term stability was assessed by pipetting $80 \mu$ of a randomly selected urine sample onto each of 12 replicate swabs and inserting them carefully in a tube with a desiccant stopper for storage at ambient temperature over a period of up to 566 days. One or more DUS was retrieved after 1, 8, 23, 36, 60, 96 or 566 days and extracted in $1 \mathrm{ml}$ of milli-Q water and the analytical response of each metabolite was obtained by UHPLC-MS/MS to investigate whether the signals were still above LOD. No further quantitation via external calibration was conducted.

\section{Urine postdilution \& DUS postextraction recovery}

For a spiking experiment six randomly selected urine samples were used undiluted for preparing DUS extracts or after $10 \times$ dilution as wet urine samples. Both diluted urine samples and DUS extracts were spiked with high and low concentration levels of calibrant (Supplementary Table 3). All nonspiked (nominal) and spiked samples were analyzed to calculate the recovery separately for diluted urine and DUS extracts according to the following formula: Recovery $=\mathrm{c}$ (spiked sample or DUS extract) $/ \mathrm{c}$ (nonspiked sample or nonspiked DUS extract) $\times 100 \%$. Recoveries found in diluted urine were compared with recoveries in DUS extracts by two-tailed heteroscedastic t-test (RStudio ver. 1.0.136, RStudio, Inc., MA, USA). 
Figure 2. Individual total daily creatinine excretion in 26 subjects measured in $24 \mathrm{~h}$ urine measured by the standard bioanalytical assay. Error bars represent standard deviation of seven measurements (6 for subject \#23).

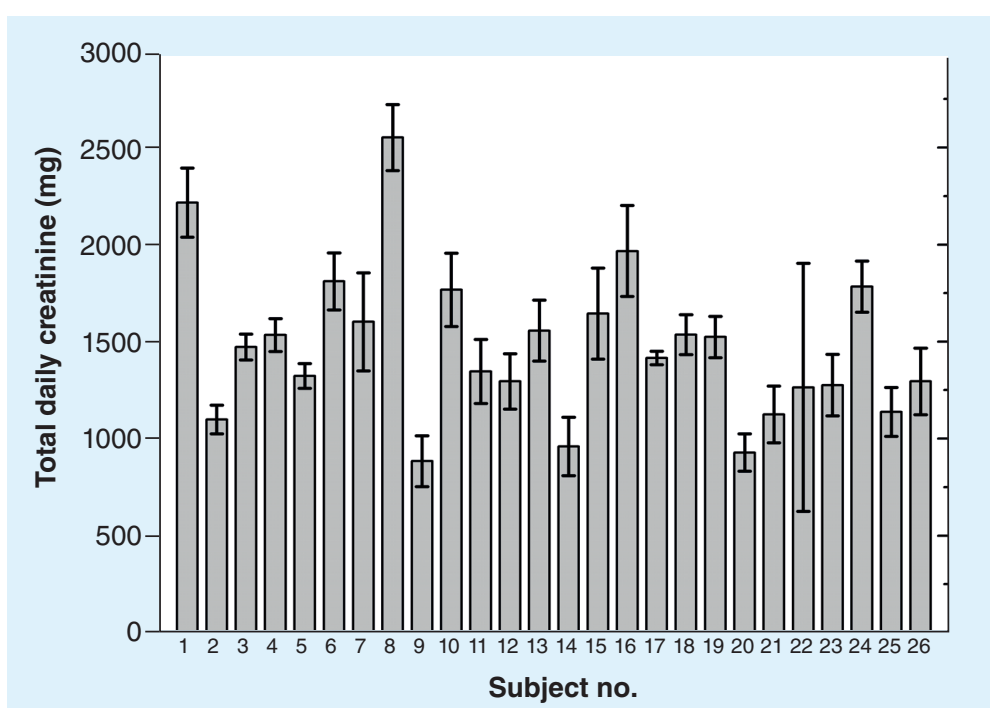

\section{Statistical analysis}

The measured total daily excretions of target analytes in $24 \mathrm{~h}$ urine $(\mathrm{X})$ and DUS (Y) were compared by PassingBablok regression in MedCalc Statistical Software (ver. 17.2, MedCalc Software, Ostend, Belgium) and RStudio (using package ' $m c r$ '). Bland-Altman unit difference plots for evaluation of the presence of outliers were done in MedCalc Statistical Software and Sigma Plot 13.0 (Systat Software, Inc., CA, USA). Graphical presentation of creatinine measurements was done in OriginPro 9.1 (OriginLab Corporation, MA, USA).

\section{Results \& discussion}

\section{Creatinine measurements}

The creatinine levels in $24 \mathrm{~h}$ urines and DUS were measured for two purposes. First, the creatinine values were used for calculating the correction factor ' $f$ ' (see Figure 1 and the 'Creatinine analysis' section) for each individual DUS sample and second, we wanted to investigate the intrapersonal variation of creatinine levels across the trial period (6 weeks). This is important for estimating the possible error in case the concept presented here will be applied in long-term trials where the average individual creatinine excretion obtained from the minimum number of $24 \mathrm{~h}$ urines would be used for the correction of DUS results. As illustrated in Figure 2, the total daily individual creatinine excretion in seven $24 \mathrm{~h}$ urine samples varied (expressed as CV\%) from 2.5 to $50.5 \%(\mathrm{n}=26)$. One subject (\#22) delivered two $24 \mathrm{~h}$ urine samples of less than $70 \mathrm{ml}$, while all others were larger than $500 \mathrm{ml}$, indicating lack of compliance or intermittent severe dehydration. After omitting this subject, the average $\mathrm{CV} \%$ of creatinine excretion was $9.8 \pm 3.7 \%$ (range $2.5-15.5 \%, \mathrm{n}=25$ ). The $\mathrm{CV} \%$ value for subject number 23 was calculated out of 6 collections since on one occasion the subject failed to deliver a $24 \mathrm{~h}$ urine. All measurements of the quality control samples $(n \geq 6)$ had $C V \leq 3 \%$. These findings suggest that the average personal creatinine value could be used as an individual correction factor if ca. 10\% error would be acceptable for the purpose of the particular study. However, this finding has to be repeated and investigated further in larger population groups as well as in different patient groups with good control that urine collections are complete. We and others have previously used parahydroxybenzoic acid as a marker of complete urine excretion; however, the variation accepted with this method is typically $\pm 15 \%$ and even larger in the elderly [23]. This is similar to or higher than the uncertainty that we observe here using individual creatinine excretion values and only studies using metabolic wards or hospitalized patients would therefore provide sufficient assessment of complete urine collection.

In this study, we did not control for incomplete urine volume collection using parahydroxybenzoic acid, therefore the variations may be caused by both the natural excretion variation as well as incomplete collections. Garde et al. [24] summarized controlled studies with relatively heterogeneous groups investigating within-individual variation of creatinine daily excretion rates. Within-individual variation across numerous studies was in the range 9-35\% which is in agreement with our findings. 
Relative extraction recovery, repeatability \& stability of DUS

Additional experiments were done in order to possibly uncover the cause of uncertainties observed in DUS extractions of different compounds. The crucial aspect of the presented research is whether the extraction efficiency of creatinine and other analytes will be comparable across all chemical classes within the same DUS since a common creatinine correction factor is used for all analytes in each sample. A detailed overview of the extraction efficiency is provided in Supplementary Tables 3 and 4. Average relative extraction recovery after $15 \mathrm{~min}$ was found to be in the range $27-52 \%$. However, for creatinine it was approximately $117 \%$. After removing one outlier $(77.77 \%$ relative extraction recovery for tartaric acid), it was found that the average CV\% for relative extraction recovery of all analytes within the same DUS was in the range 9-19\%. This indicates that metabolites having different structures and polarity may still tend to desorb from the swab in a consistent way. Even immediate extraction of wet DUS resulted in a relative recovery below approximately $60 \%$, indicating that there could be a deep compartment in the cotton material from which analytes are not extractable by water. It remains unclear why the extraction efficiency was lower for all analytes compared with creatinine. Longer extraction time (Supplementary Table 4) or further extraction with addition of methanol did not lead to additional efficacy; the relative recovery in the second extracts made in 20\% methanol was found to be maximally $13 \%$ (data not included in Supplementary Table 4) of the first extract which would correspond mostly to the transferred carry-over from the wet DUS.

Repeatability $(\mathrm{n}=8)$ of the extraction procedure (Supplementary Table 5) was found with an average CV\% of $12.7 \pm 6.9 \%$. It was lowest for creatinine $(7.3 \%)$ and highest $(\approx 29 \%)$ for EtG. For comparison, the average CV\% for repeatability $(\mathrm{n}=8)$ of the UHPLC-MS/MS analysis of urines used for preparing DUS and as nominals for calculating the relative recovery was $6.9 \pm 9.2 \%$; best for CS $(1.5 \%)$ and worst $(\approx 27 \%)$ for EtG indicating that the analytical method was the major source of error for EtG. The levels of EtG in urine and DUS extract were borderline in respect to LLOQ and therefore higher $\mathrm{CVs} \%$ were expected based on the method validation published earlier [19]. Prolonged extraction time up to $60 \mathrm{~min}$ compared with $15 \mathrm{~min}$ seemed to only slightly increase relative recovery by about $5 \pm 2 \%$ on average.

Long-term stability tests (Supplementary Table 6) show that all compounds (except PgP and creatinine which were not analyzed at the earlier time points) provided analytical responses well above the LLOQ previously established for urine, even after 566 days of storage at ambient temperature. However, external calibration standards were not prepared at all time points, so we cannot objectively compare quantitative analytical responses from DUS extracts over the period of 1.5 year on the LC-MS/MS platform. Therefore, these results provide only qualitative information about long-term storage capability of DUS. Additional studies using quantitative analysis would be required to estimate any true loss as a function of time.

\section{Urine postdilution \& DUS postextraction recovery}

In order to examine whether the accuracy of the UHPLC-MS/MS method can be affected by DUS extract matrix compared with regular urine samples a series of recovery experiments was performed. As clearly shown in Supplementary Table 3, there was no difference in average recovery values of these two matrices except possibly for low-level spiked InS. Therefore, we exclude the possibility that the analytical method might consistently perform differently in DUS extracts and urine.

\section{Qualitative comparison \& agreement between $24 \mathrm{~h}$ urine \& DUS samples}

Quality control urine samples were included to ensure correctness of the analytical process. A set of four different pooled urine samples was each consecutively injected after every 10 study samples as a control for uniformity within and between batch analyses (urine and DUS sets of the 181 samples in total on six plates) and the CV\% for the particular plate as well as for all total pools were calculated for all targeted compounds. Quality control results are summarized in Table 1. Linearity of the calibration curves was achieved with coefficients of determination $\mathrm{r}^{2}>0.99$ in all cases. The back calculated concentrations of the calibration standards met the generally accepted bioanalytical guideline criteria, where the back-calculated calibrant concentrations should be within $\pm 15 \%$ of the nominal value, except for the LLOQ for which it should be within $\pm 20 \%$; at least $75 \%$ of the calibration standards, with a minimum of six calibration standard levels, must fulfill these criteria, in our case they all did. Calculated CVs\% of total pools (of all samples) confirmed consistency of the analytical procedure for all six plates analyzed during 6 days. Qualitative assessment was evaluated based on a simple principle, in other words, whether the metabolite was detected or not comparing DUS and 24 urine analytical data for each sample. This is particularly important for $\mathrm{EtS}$ and $\mathrm{EtG}$, which were detected only in $50 \%$ of the samples. In terms of this qualitative assessment, there was 


\begin{tabular}{|c|c|c|c|c|c|c|c|}
\hline Quality control & Tartaric acid & Ethyl sulfate & $\begin{array}{l}\text { Ethyl } \\
\text { glucuronide }\end{array}$ & Indoxyl sulfate & Cresol sulfate & $\begin{array}{l}\text { Pyroglutamyl } \\
\text { proline }^{\dagger}\end{array}$ & DHEAS \\
\hline Linear range $\left(\mu \mathrm{g} \mathrm{ml}^{-1}\right)$ & $0.064-4.1$ & $0.030-0.50$ & $0.04-2.5$ & $0.15-9.9$ & $0.080-20$ & $0.040-10.2$ & $0.00065-2.7$ \\
\hline Calibration slopes CV\% $(n=6)$ & 5.8 & 8.6 & 10.4 & 3.11 & 9.1 & 2.9 & 3.9 \\
\hline \multicolumn{8}{|l|}{ 24-h urine samples } \\
\hline $\begin{array}{l}\text { Plate pools CV\% range ( } n=8 \text { per } \\
\text { batch) }\end{array}$ & $7.3-9.3$ & $13-34$ & $\ddagger$ n.a. & $3.0-5.4$ & $1.0-2.2$ & $2.8-7.1$ & $1.0-1.5$ \\
\hline $\begin{array}{l}\text { Spiked plate pools CV\% range } \\
\text { ( } n=8 \text { per batch) }\end{array}$ & $12-16$ & $13-23$ & $29-49$ & $13-16$ & $12-18$ & $6.1-17$ & $6.1-14$ \\
\hline Total pools CV\% $(n=23)$ & 9.9 & 20 & $\ddagger$ n.a. & 5.7 & 9.3 & 5.2 & 2.4 \\
\hline Spiked total pool CV\% $(n=22)$ & 5.5 & 12 & 29 & 5.2 & 6.9 & 5.6 & 1.5 \\
\hline \multicolumn{8}{|l|}{ Dried urine swabs } \\
\hline $\begin{array}{l}\text { Plate pools } \mathrm{CV} \% \text { range ( } \mathrm{n}=8 \text { per } \\
\text { batch) }\end{array}$ & $4.0-5.9$ & $15-31$ & $\ddagger$ n.a. & $4.1-5.0$ & $2.2-3.7$ & $5.5-9.3$ & $0.52-0.92$ \\
\hline $\begin{array}{l}\text { Spiked plate pools } \mathrm{CV} \% \text { range } \\
\text { ( } \mathrm{n}=8 \text { per batch) }\end{array}$ & $3.5-5.9$ & $8.8-16$ & $28-37$ & $4.8-7.5$ & $2.3-6.7$ & $9.6-12$ & $0.88-2.0$ \\
\hline Total pools CV\% $(n=23)$ & 6.2 & 19 & $\ddagger$ n.a. & 3.7 & 6.9 & 9.4 & 1.9 \\
\hline Spiked total pool CV\% $(n=22)$ & 10 & 11 & 25 & 4.3 & 9.5 & 12 & 2.5 \\
\hline \multicolumn{8}{|c|}{$\begin{array}{l}\text { For quality control, each plate also contained a batch pool and a total pool of all urine samples with and without spiking, injected after every } 10 \text { study samples. Spiking of the poo } \\
\text { was done by adding a calibrant mixture, selecting the mid-range concentration within the observed linear range for each compound. This was done in order to cover quality contr } \\
\text { also for samples with higher concentrations of the analytes and to cover compounds present in the unspiked pools at concentrations below the LLOQ. A set of four different poole } \\
\text { urine samples was each consecutively injected after every } 10 \text { study samples as a control for uniformity within and between batch analyses ( } 181 \text { urine samples and } 181 \text { dried urir } \\
\text { swab extracts analyzed together in randomized order in six batches) and the CVs\% for the particular plate as well as for total pools were calculated for all targeted compounds. } \\
\dagger 3 \text {-nitro tyrosine was used as a standard. } \\
\ddagger \text { The concentration levels after pooling were too low for accurate quantification. }\end{array}$} \\
\hline
\end{tabular}

no difference between DUS and $24 \mathrm{~h}$ urine analyses. Suitability of DUS for screening analysis was confirmed also for HPV testing [14], diagnosis of glutaric aciduria type 1 [3] or cytomegalovirus in newborns [8]. Other suggested applications may include compliance control in nutritional or clinical trials or presence/absence of a drug in forensics.

Quality control

Correlation analysis only examines the association between two variables, not their absolute agreement [25]. Therefore, Passing-Bablok regression [26] and Bland-Altman [27,28] graphical plots applying a confidence level of $95 \%$ were used. Only a few studies investigating the potential of DUS for quantitative analysis have applied these approaches $[4,5,29]$, while others graphically compared the expected with the observed DUS measurements $[6,8]$ or simply listed the DUS results and results obtained by the reference methodology in a table without further statistical analysis $[3,10,18]$.

We applied Passing-Bablok and Bland-Altman statistical analyses in order to examine agreement of measurements of DUS extracts after correction for creatinine (factor ' $\mathrm{f}$ ') and conventional $24 \mathrm{~h}$ urine samples using the same UHPLC-MS/MS method. In order to objectively investigate the bioanalytical potential of the concept presented in this work, it was necessary to limit possible errors, which could occur if subjects would be asked to prepare DUS themselves. Therefore, the preparation of DUS was done in situ using previously collected $24 \mathrm{~h}$ urines.

Table 2 summarizes results of Passing-Bablok regression and Bland-Altman analysis. Graphical results of these tests are shown in Supplementary Figure 6 and Supplementary Figure 7. The 95\% CI of the calculated slope and intercept by Passing-Bablok analysis were used to determine whether there is only a chance difference between the slope being equal to 1 and the intercept being equal to zero, indicating agreement. There were no differences between Passing-Bablok regression results obtained in MedCalc and Rstudio. Taking into account the slopes obtained by Passing-Bablok regression, DUS results were underestimated compared with $24 \mathrm{~h}$ urines in case of $\mathrm{TaA}, \mathrm{InS}, \mathrm{PgP}$ and DHEAS, while partially overestimated for CrS. In case of EtG and EtS, the statistical analysis showed apparent agreement (no deviation from unity) between DUS and $24 \mathrm{~h}$ urine both for intercept and slopes, respectively. However, there was a proportional bias for higher concentrations of several analytes leading to an overall large standard deviation in the Bland-Altman plots. For some applications, such as precise quantification of $\mathrm{EtG}$ for immediate alcohol consumption control the urine swab would therefore not give correct estimates, 
Table 2. Passing-Bablok regression and Bland-Altman analysis comparing analyses of seven compounds in 181 urine samples and 181 dried urine swab extracts.

\begin{tabular}{|c|c|c|c|c|c|c|}
\hline \multirow[t]{2}{*}{ Analyte } & \multicolumn{2}{|c|}{ Passing Bablok } & \multirow{2}{*}{$\begin{array}{l}\text { CUSUM test } \\
\text { Linearity }\end{array}$} & \multirow{2}{*}{$\begin{array}{l}\text { Spearman rank } \\
\text { Correlation } \\
\text { co-efficient } 95 \% \mathrm{Cl} \\
(p<0.0001)\end{array}$} & \multicolumn{2}{|c|}{ Bland-Altman } \\
\hline & Intercept $95 \% \mathrm{Cl}$ & Slope $95 \% \mathrm{Cl}$ & & & $\begin{array}{l}\text { Number of } \\
\text { outliers/total } \\
\text { number of samples }\end{array}$ & $\mathrm{p}\left(\mathrm{H}_{0}:\right.$ mean $\left.=0\right)$ \\
\hline Tartaric acid & -0.92 to -0.37 & $0.79-0.88$ & $p=0.86$ & $0.95-0.97$ & $8 / 181$ & $<0.0001$ \\
\hline Ethyl sulfate & $-60-18$ & $1.0-1.3$ & $p=0.13$ & $0.87-0.94$ & $2 / 90$ & 0.74 \\
\hline Ethyl glucuronide & $-340-230$ & $0.76-1.2$ & $p=0.41$ & $0.70-0.89$ & $1 / 65$ & 0.15 \\
\hline Indoxyl sulfate & $-1.1-2.9$ & $0.80-0.89$ & $p=0.22$ & $0.89-0.93$ & $5 / 181$ & $<0.0001$ \\
\hline Cresol sulfate & -9.8 to -0.88 & $1.1-1.2$ & $p=0.05$ & $0.85-0.92$ & $13 / 181$ & $<0.0001$ \\
\hline Pyroglytamyl proline & $-1.0-0.71$ & $0.79-0.89$ & $p=0.22$ & $0.72-0.84$ & $8 / 181$ & $<0.0001$ \\
\hline DHEAS & $0.92-7.8$ & $0.77-0.80$ & $p=0.22$ & 0.99 & $7 / 181$ & $<0.0001$ \\
\hline
\end{tabular}

especially after higher alcohol intakes. CUSUM (cumulative sum control chart) test did not show any significant deviation from linearity for any of the metabolites. Spearman correlation rank was $>0.9$ within the defined CI $(\alpha=0.05)$ in all cases, except for TaA and PgP, indicating overall very good correlation between DUS and wet urine analyses. Especially for DHEAS the high correlation value of 0.99 illustrates that this can be misleading when evaluating agreement between the two sets of measurements.

Bland-Altman analysis requires normally distributed data; however, none of the differences between the measurements followed the normal distribution. Logarithmic or square root transformations did not help in providing normal distributions, so Bland-Altman results have to be considered as indicative only. Bland-Altman analysis indicated that the number of outliers varied from $1.5 \%(\mathrm{EtG})$ to $7.2 \%(\mathrm{CrS})$ of the total number of measurements; however, this is affected by the non-normal distribution and by the residual bias observed for several analytes indicating nonlinearity of concentration dependent adsorption to DUS for several metabolites. For all analytes, except $\mathrm{EtS}$ and $\mathrm{EtG}$ we had to reject the $\mathrm{H}_{0}$ hypothesis claiming that the difference between two sets of measurements is equal to 0 . This is in support of our Passing-Bablok regression findings. The sample size in case of EtS and EtG was lower compared with the other analytes since these metabolites are present only after alcohol intake, but alcohol was not consumed by the subjects on all collection days.

There may be several possible causes of the observed disagreements between the two sampling techniques studied in this work. In order to allow DUS to predict $24 \mathrm{~h}$ urine levels after creatinine adjustment, the compounds should have similar affinity toward the cotton swab and the same stability as creatinine, and they should have the same stability in frozen urine as they have on dried cotton swabs at room temperature. Moreover, the loss from contact of swabs with the plastic tube should be minimized in order to maintain the concentration of metabolites in the extract above their LLOQ. Regarding affinity to the cotton swab this factor would be expected to be negligible during soaking if the urine is displacing air by capillary effects rather than actually being adsorbed onto the cotton. Our experiments show that higher extraction efficiency was achieved for creatinine than for the tested metabolites. Therefore, stronger adsorption of the other compounds could be an explanation for underestimation of the results. Moreover, the calculated dilution factor (raw data in Supplementary Table 4) from creatinine concentration measured in urine/concentration in the DUS was found on average to be $10.8 \pm 1.1$, while the theoretical dilution assuming $100 \%$ recovery was 12.5 . The number of outliers differs among compounds as well as regression analysis parameters, again indicating the presence of a nonsystematic error. This is additionally supported by Bland-Altman graphs where the distribution of points differs among parameters and the bias is not consistent but somewhat concentration dependent for most analytes, showing lower extraction efficiencies at higher concentrations. Last but not least, the recovery from the swab might vary with extraction time too although no change was observed with an increase from 20 to 60 min but even longer times might have increased recovery. Differential extraction efficiency with CVs up to $19 \%$ for the target compounds could explain these nonsystematic errors. Further experiments with additional adsorbents would be needed to explain the reproducible, yet incomplete extraction of most analytes from the cotton swabs after drying. It is also reasonable to propose minimizing the extraction volume to obtain creatinine levels in DUS extracts closer to the concentrations measured in undiluted urine since DUS creatinine values were around its LLOQ which could introduce additional errors. Alternatively, swabs with higher capacity 
could be used since the extracted amounts available for analysis would affect the available number of analytes above LOD or LLOQ.

The storage time of the DUS varied from 5 to 9 months and there is limited literature describing such a long-term stability of compounds in DUS, for example, the storage stability of EtS and EtG was tested only for 3 weeks while urine was spiked with $E$. coli [30]. It is reasonable to expect good stability of the analytes when water activity is low and compounds are protected from light; these are common storage conditions of stock crystalized or powdered chemicals, storable for years. The drying process of the swab head in the DUS kit used in this work took up to $48 \mathrm{~h}$ and it can be considered as the most critical period for sample degradation. Hypothetically, certain bacterial or oxidative degradation could occur within this period but bench top stability tests (samples were stored at $5^{\circ} \mathrm{C}$ for 15-20 h in the LC sample manager) performed within the method validation [19] did not show any compound degradation in liquid samples. However, loss may happen while water activity is declining below critical values when metabolites would be in soluble form to permeate the cell wall in case of microbial contamination [31]. On the other hand, microbial degradation would be highly variable between samples and would not seem plausible with the fair reproducibility for the analytes observed here. Loss of compounds due to uneven desorption after contact with the plastic wall of the collection tube could also be the cause of some loss. Finally and most importantly, the extraction loss was observed to be immediate and independent of drying. Our findings therefore point to unextracted residuals as the most plausible cause of discrepancies between frozen urine storage and DUS stored at room temperature while loss during drying may add some additional variation. Since drying of the cotton swab inside the plastic tube can take up to $48 \mathrm{~h}$, speeding up the drying process by improving the collection kit design should be considered. The relatively consistent changes for all compounds, except creatinine and the low number of outliers indicate that DUS could be a useful alternative to wet urine collection in larger studies. Further metabolomics investigations would be needed in order to find out whether the extraction loss is similar for most compounds or not.

The strengths of our DUS approach are that the method provides simple one-step sampling and convenient sample delivery to the laboratory for volunteers. The method also provides results proportional to the actual values for each of the tested analytes, albeit not quantitative. Finally, the DUS storage is much less demanding on electrical energy than usual urine storage in a freezer. The weaknesses are that the method is yet neither accurate nor precise for the analytes and would need correction for the estimated loss of each compound analyzed. Moreover, the space requirements for storage compared with, for example, urine spots on paper cards may be seen as a disadvantage.

\section{Conclusion}

UHPLC-MS/MS analysis of various human metabolites in DUS, including results correction via creatinine ratios was reliable for qualitative (screening) analysis but underestimated quantitative results by up to $11-23 \%$ compared with measurements in conventional $24 \mathrm{~h}$ urine samples. We assume this was mostly due to higher extraction efficiency for creatinine than for the metabolites. Bias proportional to concentration was observed in case of alcohol intake related metabolites, while cresol sulfate was overestimated by $10-20 \%$. Therefore, we do not conclude that the dried urine swab sampling technique on cosmetic swabs is a fully reliable replacement for $24 \mathrm{~h}$ urine samples for quantitative measurements unless the correction for the offset can be determined or the bias is within an acceptable range for the research conducted. However, due to the ease of DUS sampling and considering the common lack of compliance when volunteers collect $24 \mathrm{~h}$ urines, we think that the error may in practice be comparable between the two sampling techniques. This study also examined individual variance of daily total creatinine excretion, which was below $16 \%$. This finding has an important implication for future studies when creatinine correction factors could be determined as an average individual value from $24 \mathrm{~h}$ urine samples.

\section{Future perspective}

In clinical trials, microvolume urine spotting on cotton swabs may be a simpler and convenient sampling technique for subjects than urine collection in flasks or making DUS on filter paper cards. Although, electrical energy requirements are null for dried storage the space requirements for DUS are currently disadvantageous compared with paper cards. Although, the dried urine spotting technique seems very promising more methodological studies have to be conducted in order to improve applicability of dried urine microvolume fluidic sampling. 
Summary points

Background

- We tested a large set $(n=181)$ of dried urine samples spotted on regular cosmetic cotton swabs for quantitative UHPLC-MS/MS analysis of various metabolites across a wide polarity and structural range.

Experimental

- The agreement of measurements between conventional $24 \mathrm{~h}$ urines and dried urine spots made from them in situ was evaluated by Passing-Bablok regression and Bland-Altman analysis after creatinine correction.

Results \& discussion

- There was full agreement in qualitative results making dried urine spots a simple method for urine sample collection, suitable for metabolomics profiling and for screening of compliance in clinical trials.

- Quantitative analysis revealed that results were underestimated in the range from 11 to $23 \%$ in case of tartaric acid, indoxyl sulfate, pyroglutamyl proline and DHEAS. Better agreement was found in case of the alcohol intake related metabolites, ethyl sulfate and ethyl glucuronide but with a proportional bias at higher concentrations leading to underestimation. Partial overestimation ranging from 10 to $20 \%$ was observed in case of cresol sulfate.

- As a secondary objective, we examined variance of individual total daily creatinine excretion, which was found to be maximally $16 \%$.

- The dried urine samples contained analytes at measurable levels for at least 9 months.

Conclusion

- Our findings suggest that the individual creatinine correction factor could be used in long-term clinical trials for correction of measurements in dried urine spots, provided that muscle mass is unchanged and the level of analytical offset is acceptable for the research purpose.

- The dried urine swab samples can be stored at room temperature and analyzed later, thereby making logistics much easier in human studies.

project number CF14-0985 'Metabolic Imprints of Alcoholic Beverages' (MetAl) in order to support preceding work on the clinical trial which provided samples used in this work.

\section{Ethical conduct of research}

The study protocol was approved by the municipal Ethical Committee of the capital region of Denmark (journal number: $\mathrm{H}-$ 15016961) in accordance with the Helsinki-II declaration. The study was registered on Clinicaltrials.gov (NCT03384147). Before entering the study, all participants gave their written consent after having received written and oral information about the study.

Supplementary data

To view the supplementary data that accompany this paper please visit the journal website at: www.futurescience.com/doi/suppl/10.4155/bio-2018-0042

\section{Open access}

This work is licensed under the Attribution-NonCommercial-NoDerivatives 4.0 Unported License. To view a copy of this license, visit http://creativecommons.org/licenses/by-nc-nd/4.0/

\section{References}

Papers of special note have been highlighted as: • of interest; $\bullet \bullet$ of considerable interest

1. DBS Database. www.spotonsciences.com/dbs-database

2. Wilhelm AJ, Den Burger JC, Swart EL. Therapeutic drug monitoring by dried blood spot: progress to date and future directions. Clin. Pharmacokinet. 53(11), 961-973 (2014).

3. Al-Dirbashi OY, Kolker S, Ng D et al. Diagnosis of glutaric aciduria type 1 by measuring 3-hydroxyglutaric acid in dried urine spots by liquid chromatography tandem mass spectrometry. J. Inherit. Metab. Dis. 34(1), 173-180 (2011).

4. Antunes MV, Niederauer CG, Linden R. Development, validation and clinical evaluation of a dried urine spot method for determination of hippuric acid and creatinine. Clin. Biochem. 46(13-14), 1276-1280 (2013).

5. Carreño Balcázar JS, Meesters RJW. Bioanalytical comparison between dried urine spots and liquid urine bioassays used for the quantitative analysis of urinary creatinine concentrations. Bioanalysis 6(21), 2803-2814 (2014).

- Creatinine levels were compared in urine and dried urine spot samples.

6. Ohira S-I, Kirk AB, Dyke JV, Dasgupta PK. Creatinine adjustment of spot urine samples and $24 \mathrm{~h}$ excretion of iodine, selenium, perchlorate, and thiocyanate. Environ. Sci. Technol. 42(24), 9419-9423 (2008). 
- Creatinine adjustment of spot urine samples and $24 \mathrm{~h}$ excretion was used for of iodine, selenium, perchlorate, and thiocyanate determination.

7. Barcenas M, Suhr TR, Scott CR, Turecek F, Gelb MH. Quantification of sulfatides in dried blood and urine spots from metachromatic leukodystrophy patients by liquid chromatography/electrospray tandem mass spectrometry. Clin. Chim. Acta 433, 39-43 (2014).

8. Forman M, Valsamakis A, Arav-Boger R. Dried urine spots for detection and quantification of cytomegalovirus in newborns. Diagn. Microbiol. Infect. Dis. 73(4), 326-329 (2012).

9. Michely JA, Meyer MR, Maurer HH. Dried urine spots - a novel sampling technique for comprehensive LC-MSn drug screening. Anal. Chim. Acta 982(Supplement C), 112-121 (2017).

10. Otero-Fernandez M, Cocho JA, Tabernero MJ, Bermejo AM, Bermejo-Barrera P, Moreda-Pineiro A. Direct tandem mass spectrometry for the simultaneous assay of opioids, cocaine and metabolites in dried urine spots. Anal. Chim. Acta 784, 25-32 (2013).

11. Lee Y, Lai KK, Sadrzadeh SM. Simultaneous detection of 19 drugs of abuse on dried urine spot by liquid chromatography-tandem mass spectrometry. Clin. Biochem. 46(12), 1118-1124 (2013).

12. Kummer N, Lambert WE, Samyn N, Stove CP. Alternative sampling strategies for the assessment of alcohol intake of living persons. Clin. Biochem. 49(13-14), 1078-1091 (2016).

13. Suh JH, Eom HY, Kim U et al. Highly sensitive electromembrane extraction for the determination of volatile organic compound metabolites in dried urine spot. J. Chromatogr. A 1416 1-9 (2015).

14. Frati ER, Martinelli M, Fasoli E et al. HPV testing from dried urine spots as a tool for cervical cancer screening in low-income countries. Biomed. Res. Int. 2015283036 (2015).

15. Kerrigan S, Specimen selection considerations. In: Clarkés Analytical Forensic Toxicology. Negrusz A, Cooper GAA (Eds). Pharmaceutical Press, London, UK, 335-356 (2013).

16. Holland NT, Smith MT, Eskenazi B, Bastaki M. Biological sample collection and processing for molecular epidemiological studies. Mutat. Res. Rev. 543(3), 217-234 (2003).

17. Resano M, Aramendía M, Rello L, Calvo ML, Bérail S, Pécheyran C. Direct determination of Cu isotope ratios in dried urine spots by means of fs-LA-MC-CPMS. Potential to diagnose Wilson's disease. J. Anal. At. Spectrom. 28(1), 98-106 (2013).

18. Rello L, Lapeña AC, Aramendía M, Belarra MA, Resano M. A dried urine spot test to simultaneously monitor Mo and Ti levels using solid sampling high-resolution continuum source graphite furnace atomic absorption spectrometry. Spectrochim. Acta B 81, 11-19 (2013).

19. Monosik R, Dragsted LO. A versatile UHPLC-MSMS method for simultaneous quantification of various alcohol intake related compounds in human urine and blood. Anal. Method 8(38), 6865-6871 (2016).

-• Detailed description of analytical method used in this study.

20. Pierson-Perry JF, Vaks JE, Durham AP et al. Evaluation of detection capability for clinical laboratory measurement procedures; approved guideline. (2nd Edition). Clinical and Laboratory Standards Institute, PA, USA, 32, 80 (2012).

21. Stanstrup J, Rasmussen JE, Ritz C, Holmer-Jensen J, Hermansen K, Dragsted LO. Intakes of whey protein hydrolysate and whole whey proteins are discriminated by LC-MS metabolomics. Metabolomics 10(4), 719-736 (2014).

22. Gurdeniz G, Jensen MG, Meier S, Bech L, Lund E, Dragsted LO. Detecting beer intake by unique metabolite patterns. J. Proteome Res. 15(12), 4544-4556 (2016).

23. Leclercq C, Maiani G, Polito A, Ferro-Luzzi A. Use of PABA test to check completeness of 24-h urine collections in elderly subjects. Nutrition 7(5), 350-354 (1991).

-. Compares uncertainties related to standardization of urine samples with volume and creatinine concentration.

24. Garde AH, Hansen ÅM, Kristiansen J, Knudsen LE. Comparison of uncertainties related to standardization of urine samples with volume and creatinine concentration. Ann. Occup. Hyg. 48(2), 171-179 (2004).

25. Giavarina D. Understanding Bland Altman analysis. Biochem. Med. 25(2), 141-151 (2015).

26. Passing H, Bablok W. A new biometrical procedure for testing the equality of measurements from two different analytical methods. Application of linear regression procedures for method comparison studies in clinical chemistry, Part I. J. Clin. Chem. Clin. Biochem. 21(11), 709-720 (1983).

27. Bland JM, Altman DG. Statistical methods for assessing agreement between two methods of clinical measurement. Lancet 1(8476), 307-310 (1986).

28. Bland JM, Altman DG. Statistical methods for assessing agreement between two methods of clinical measurement. Int. J. Nurs. Stud. 47(8), 931-936 (2010).

29. Gonzalez D, Melloni C, Poindexter BB et al. Simultaneous determination of trimethoprim and sulfamethoxazole in dried plasma and urine spots. Bioanalysis 7(9), 1137-1149 (2015).

30. Redondo AH, Korber C, Konig S, Langin A, Al-Ahmad A, Weinmann W. Inhibition of bacterial degradation of EtG by collection as dried urine spots (DUS). Anal. Bioanal. Chem. 402(7), 2417-2424 (2012). 
31. Zhang L, Sun DW, Zhang Z. Methods for measuring water activity (aw) of foods and its applications to moisture sorption isotherm studies. Crit. Rev. Food Sci. Nutr. 57(5), 1052-1058 (2017). 